\title{
Large-area [Fe II] Line Mapping of the Supernova Remnant IC 443 with the IRSF/SIRIUS
}

\author{
Takuma Kokusho ${ }^{1}$, Takahiro Nagayama ${ }^{1}$, Hidehiro Kaneda ${ }^{1}$, \\ Daisuke Ishihara $^{1}$, Ho-Gyu Lee ${ }^{2}$, and Takashi Onaka ${ }^{2}$ \\ kokusho@u.phys.nagoya-u.ac.jp
}

Received —

\footnotetext{
${ }^{1}$ Graduate School of Science, Nagoya University, Chikusa-ku, Nagoya 464-8602, Japan

${ }^{2}$ Department of Astronomy, Graduate School of Science, The University of Tokyo, Bunkyo-ku, Tokyo 113-0033, Japan
} 


\begin{abstract}
We present the result of near-infrared (near-IR) [Fe II] line mapping of the supernova remnant IC 443 with the IRSF/SIRIUS, using the two narrow-band filters tuned for the [Fe II] $1.257 \mu \mathrm{m}$ and [Fe II] $1.644 \mu \mathrm{m}$ lines. Covering a large area of $30^{\prime} \times 35^{\prime}$, our observations reveal that $[\mathrm{Fe}$ II] filamentary structures exist all over the remnant, not only in an ionic shock shell, but also in a molecular shock shell and a central region inside the shells. With the two [Fe II] lines, we performed corrections for dust extinction to derive the intrinsic line intensities. We also obtained the intensities of thermal emission from the warm dust associated with IC 443, using the far- and mid-IR images taken with $A K A R I$ and Spitzer, respectively. As a result, we find that the [Fe II] line emission relative to the dust emission notably enhances in the inner central region. We discuss causes of the enhanced [Fe II] line emission, estimating the $\mathrm{Fe}^{+}$and dust masses.
\end{abstract}

Subject headings: infrared: ISM — ISM: individual objects (IC 443) — ISM: supernova remnants 


\section{Introduction}

IC 443 is a Galactic supernova remnant (SNR) with an age of 3000-30000 years (Petre et al. 1988; Olbert et al. 2001). Existence of a pulsar wind nebula (Gaensler et al. 2006) and metal-rich X-ray plasma (Troja et al. 2008) indicates that the SNR is of a core-collapse origin. IC 443 shows a limb-brightened morphology at optical, infrared, and radio wavelengths (Bykov et al. 2008; Castelletti et al. 2011), while it shows a centrally-peaked morphology in the X-ray (Troja et al. 2008). Hence IC 443 is recognized as a mixed-morphology-type SNR. The distance to IC 443 is estimated to be $1.5 \mathrm{kpc}$ (Welsh \& Sallmen 2003) and we use this value throughout the present paper.

One of the most interesting and impressive features of IC 443 is its heavy interaction with the interstellar medium (ISM) via ionic and molecular shocks, which makes a marked contrast between the near-infrared (near-IR) morphologies. The 2MASS survey revealed that IC 443 has a bright shell in the $J$ and $H$ bands in the northeast region, which is faint in the $K_{s}$ band. From the shell, the [Fe II] $1.644 \mu \mathrm{m}$ line emission was detected (Graham et al. 1987). Rho et al. (2001) concluded that the dominant carriers of both $J$ and $H$ bands are [Fe II] line emission. In contrast, the southern ridge is bright in the $K_{s}$ band but faint in the $J$ and $H$ bands. The near-IR spectroscopic observation with $A K A R I$ detected many ro-vibrational transition lines of $\mathrm{H}_{2}$ from parts of the southern ridge (Shinn et al. 2011 ). Therefore the $\mathrm{H}_{2}$ emission at $2.12 \mu \mathrm{m}$ is likely to dominate in the $K_{s}$ band in the southern ridge. Noriega-Crespo et al. (2009) showed that the [Fe II] $26 \mu \mathrm{m}$ line emission also exists in the spectra taken along the southern ridge with the Spitzer/IRS. Thus IC 443 is an excellent laboratory to investigate ionic and molecular shock interactions between a SNR and the ambient ISM.

This paper focuses on the spatial distribution of the [Fe II] line emission in IC 443. The origin of the [Fe II] line emission in SNRs is still controversial. Some mechanism to 
efficiently produce gas-phase Fe is required because more than $99 \%$ of $\mathrm{Fe}$ is usually depleted onto dust grains in the interstellar space (Draine 1995). A conventional idea is that the gas-phase Fe is produced by sputtering Fe-bearing dust grains, but we cannot rule out a possibility of supernova ejecta origins. Koo et al. (2007) suggested that the [Fe II] line emission in SNR G11.2-0.3 is partly of an ejecta origin, because, considering its expansion velocity, an ejecta is likely to be excited recently by a reverse shock, where the [Fe II] line was detected. To examine the origin of the [Fe II] line emission, it is important to make a detailed comparison of the spatial distribution of the [Fe II] line with that of the dust emission associated with IC 443.

\section{Observations and data reduction}

We observed IC 443 with the near-IR camera SIRIUS (Simultaneous Infrared Imager for Unbiased Survey; Nagashima et al. 1999; Nagayama et al. 2003) on the IRSF (Infrared Survey Facility) $1.4 \mathrm{~m}$ telescope. IRSF is located at the South African Astronomical Observatory (SAAO), and has been operated by Nagoya University and the SAAO. The SIRIUS camera has a field of view of $7.7 \times 7.7$ with a pixel scale of $0^{\prime \prime} 45$. We observed 44 contiguous fields toward IC 443 , which, in total, correspond to the area of $30^{\prime} \times 35^{\prime}$, using the two narrow-band filters tuned for the two [Fe II] lines at $1.257 \mu \mathrm{m}$ and $1.644 \mu \mathrm{m}$. The details of the observations are summarized in Table 1 .

We applied the standard data reduction procedure to the array images, including dark subtraction, flat-fielding, sky subtraction, and dithered-image-combining. As a result, we obtained 44 images for each of the $1.257 \mu \mathrm{m}$ and $1.644 \mu \mathrm{m}$ filters, which were combined into a large-area map, covering the main structure of IC 443. We performed photometric calibration, making comparison with the 2MASS point source catalog (PSC;

Skrutskie et al. 2006). We used the $J$ - and $H$-band magnitudes for the calibrations of the 
$1.257 \mu \mathrm{m}$ and $1.644 \mu \mathrm{m}$ filter images, respectively, assuming that the magnitude of each star is the same between the corresponding band and filter images. For the photometry, we selected stars with fluxes higher than $12.5 \mathrm{mag}$ and flux errors smaller than $0.05 \mathrm{mag}$ from the 2MASS PSC, where we used sufficiently-isolated stars to avoid problems due to star crowding. The number of the stars used in the photometric calibration is typically 15 per image, and the uncertainties of the photometric calibration coefficients are $\sim 5 \%$. Finally, we subtract point sources with SExtracter (Bertin \& Arnouts 1996) to avoid overestimating the [Fe II] line intensities due to the stellar flux.

The far-IR images of IC 443 were derived from the $A K A R I$ all-sky-survey data. The $A K A R I$ all-sky-survey was performed between May 2006 and August 2007 in the cold mission phase with liquid helium cryogen, using the Far-Infrared Surveyor (FIS; Kawada et al. 2007). The FIS has four far-IR photometric bands at central wavelengths of $65 \mu \mathrm{m}$ (N60), $90 \mu \mathrm{m}$ (WIDE-S), $140 \mu \mathrm{m}$ (WIDE-L), and $160 \mu \mathrm{m}$ (N160), which have the effective band widths of $22,38,52$, and $34 \mu \mathrm{m}$, respectively. The data were processed with the $A K A R I$ pipeline tool originally optimized for point source extraction (Yamamura et al. 2009), and then with an additional pipeline to recover large-scale diffuse emission (Doi et al. 2012). We also used the Spitzer/MIPS $24 \mu \mathrm{m}$ image of IC 443, retrieved from the Spitzer archives (http://archive.spitzer.caltech.edu). The observation of IC 443 was part of the LATTER_SE program (PI: G. Rieke). The data were taken in a scan map mode (Rieke et al. 2004) and produced by the pipeline version S18.12.0.

\section{Results}

Figure 1 shows the images of IC 443 with the [Fe II] $1.257 \mu \mathrm{m}$ and $1.644 \mu \mathrm{m}$ filters, before the removal of the point sources. As can be seen in the figure, we detect the [Fe II] filamentary structures from a wide area of IC 443. Rho et al. (2001) detected $J$ - and $H$-band 
diffuse emission with the surface brightnesses of $2.3 \times 10^{-4}$ and $1.6 \times 10^{-4} \mathrm{ergs} \mathrm{s}^{-1} \mathrm{~cm}^{-2} \mathrm{sr}^{-1}$, respectively, from a $48^{\prime \prime} \times 18^{\prime \prime}$ area in the northeast shell, centered at (R.A., Decl.) $=$ $\left(06^{\mathrm{h}} 17^{\mathrm{m}} 34.41,+22^{\circ} 52^{\prime} 55^{\prime \prime} \cdot 2\right)$. At the same position, Graham et al. (1987) detected the [Fe II] $1.644 \mu \mathrm{m}$ line intensity of $1.1 \times 10^{-4} \mathrm{ergs} \mathrm{s}^{-1} \mathrm{~cm}^{-2} \mathrm{sr}^{-1}$, and our maps show the [Fe II] $1.257 \mu \mathrm{m}$ and $1.644 \mu \mathrm{m}$ line intensities of $1.7 \times 10^{-4}$ and $1.2 \times 10^{-4} \mathrm{ergs} \mathrm{s}^{-1} \mathrm{~cm}^{-2} \mathrm{sr}^{-1}$, respectively. Thus in the northeast shell, indeed, the [Fe II] $1.257 \mu \mathrm{m}$ and the $1.644 \mu \mathrm{m}$ line emission are dominant in the $J$ and $H$ band, respectively. More importantly, the [Fe II] filaments are detected not only in the northeast shell, which is bright in the $J$ and $H$ bands, but also in other large area including the southern ridge and a central region inside the shells. For the entire remnant, Rho et al. (2001) obtained the $J$ - and $H$-band fluxes of $1.2 \times 10^{-9}$ and $6.4 \times 10^{-10} \mathrm{ergs} \mathrm{s}^{-1} \mathrm{~cm}^{-2}$, respectively, while we detected the [Fe II] 1.257 $\mu \mathrm{m}$ and $1.644 \mu \mathrm{m}$ line fluxes of $8.2 \times 10^{-10}$ and $6.2 \times 10^{-10} \mathrm{ergs} \mathrm{s}^{-1} \mathrm{~cm}^{-2}$, respectively.

To obtain the intrinsic [Fe II] line intensities, we performed corrections for foreground dust extinction, using the observed line ratio of the two [Fe II] lines at $1.257 \mu \mathrm{m}$ and 1.644 $\mu \mathrm{m}$. Since they are due to electronic transitions from the same upper level, the intrinsic line ratio must be fixed at 1.36 (Nussbaumer \& Storey 1988). We estimated the foreground extinction by comparing the observed line ratio with the theoretical one, assuming the interstellar extinction law of Cardelli et al. (1989). In calculating the line ratios, we first masked the pixels where [Fe II] $1.257 \mu \mathrm{m}$ and $1.644 \mu \mathrm{m}$ are below $1 \sigma$, because the fluctuation of background can produce strong positive or negative spikes in a ratio map. Then we smoothed the resultant ratio map with a boxcar of $150 \times 150$ pixels. The extinction map thus derived is shown in the left panel of Fig. 2 on the color scale, with the contours of the ${ }^{12} \mathrm{CO}$ map integrated in the velocity range of -10 to $0 \mathrm{~km} \mathrm{~s}^{-1}$ from Lee et al. (2012). From the figure, it can be seen that the foreground extinction is large in the west and southeast regions, and CO clouds are lying in similar regions where Troja et al. (2006) showed the absorption of X-rays. Using the extinction map, we performed the extinction corrections 
for both $1.257 \mu \mathrm{m}$ and $1.644 \mu \mathrm{m}$ images. The resultant [Fe II] $1.257 \mu \mathrm{m}$ line intensity image is shown in the right panel of Fig. 2 on the gray scale.

The far- and mid-IR images of IC 443, which are obtained by $A K A R I$ and Spitzer, respectively, include emission not only from the dust associated with IC 443, but also from foreground and background dust. Therefore discrimination of these two components is required. The dust associated with IC 443 is heated by shocks and therefore likely to have higher temperatures than foreground and background dust. We do not consider the dust component associated with IC 443, but not yet heated by shocks, if any, because it cannot be the origin of the observed $\mathrm{Fe}^{+}$through dust sputtering. Hence we fitted mid- to far-IR spectral energy distributions (SEDs) by a two-temperature modified blackbody model with the emissivity power-law index of unity. First, we fitted the model to the SED of the total region with a photometry radius of $19^{\prime}$, centered at (R.A., Decl.) $=\left(06^{\mathrm{h}} 17^{\mathrm{m}} 18.63\right.$, $\left.+22^{\circ} 37^{\prime} 07^{\prime \prime} .5\right)$. As a result, we find that the model with the temperatures of $T_{\text {cold }}=14.8$ $\mathrm{K}$ and $T_{\text {warm }}=56.3 \mathrm{~K}$ reproduces the total $\mathrm{SED}$ very well. Then with the temperatures fixed at the above best-fit values, we fitted local SEDs for every spatial bin of $15^{\prime \prime} \times 15^{\prime \prime}$, allowing only the amplitudes of the two components to vary. Here, considering possible severe contaminations of the [O I] $63 \mu \mathrm{m}$ line emission to the $65 \mu \mathrm{m}$ narrow-band (N60) intensity and [C II] $158 \mu \mathrm{m}$ line emission to the $160 \mu \mathrm{m}$ narrow-band (N160) intensity, we excluded the N60 and N160 band intensities in the local SED fitting. The contours in the right panel of Fig. 2 show the resultant distribution of the intensity of the warm dust emission integrated over the wavelength range of $2-200 \mu \mathrm{m}$, which is derived from the above SED fitting.

Here and hereafter we define regions A to D, as shown in the right panel of Fig. 2, all of which have the same area of $2.1 \times 2.1 \mathrm{pc}^{2}\left(4^{\prime} .9 \times 4^{\prime} \cdot 9\right)$. Regions A, centered at (R.A., Decl. $)=$ $\left(06^{\mathrm{h}} 17^{\mathrm{m}} 55.91,+22^{\circ} 46^{\prime} 24^{\prime \prime} .8\right)$ and D at (R.A., Decl. $)=\left(06^{\mathrm{h}} 17^{\mathrm{m}} 40^{\mathrm{s}} .58,+22^{\circ} 23^{\prime} 55^{\prime \prime} .0\right)$ represent 
the ionic shock and the molecular shock region, respectively, while regions B at (R.A., Decl. $)=\left(06^{\mathrm{h}} 17^{\mathrm{m}} 13.62,+22^{\circ} 41^{\prime} 10^{\prime \prime} 0\right)$ and $\mathrm{C}$ at $($ R.A., Decl. $)=\left(06^{\mathrm{h}} 17^{\mathrm{m}} 21^{\mathrm{s}} .75,+22^{\circ} 32^{\prime} 10^{\prime \prime} 0\right)$ represent inner central regions where the [Fe II] line emission is detected significantly. As can be seen in the figure, both [Fe II] and warm dust emission are strong in region A, while only the warm dust emission is strong in region $\mathrm{D}$. In regions $\mathrm{B}$ and $\mathrm{C}$, the [Fe II] line emission is relatively strong whereas the dust emission is faint. Near region C, Noriega-Crespo et al. (2009) also detected the [Fe II] $26 \mu \mathrm{m}$ emission in their Spitzer/IRS spectrum. More quantitatively, Fig. 3 shows the correlation plot between the intensities of the extinction-corrected [Fe II] $1.257 \mu \mathrm{m}$ line and the warm dust emission integrated over the wavelength range of $2-200 \mu \mathrm{m}$. For comparative purpose, we draw the line fitted to only the data points of region $\mathrm{A}$, which gives the slope of $0.058 \pm 0.006$ and the correlation coefficient of 0.3 . The figure clearly reveals that the [Fe II] line emission in regions $\mathrm{B}$ and $\mathrm{C}$ is notably strong as compared with the warm dust emission.

\section{Discussion}

We estimate the masses of $\mathrm{Fe}^{+}$and warm dust for regions $\mathrm{A}$ to $\mathrm{D}$. In a two-level approximation, the [Fe II] $1.644 \mu \mathrm{m}$ line intensity is given by

$$
I_{\lambda 1.644}=\frac{T_{4}^{-0.94} e^{-1.57 / T_{4}}\left(N_{\mathrm{Fe}^{+}} / 10^{16}\right)}{1+4.2 \times 10^{4} T_{4}^{0.69} / n_{e}},
$$

where $T_{4}$ is the gas temperature in units of $10^{4} \mathrm{~K}$, and $n_{e}$ and $N_{\mathrm{Fe}^{+}}$are the electron density and the $\mathrm{Fe}^{+}$column density in units of $\mathrm{cm}^{-3}$ and $\mathrm{cm}^{-2}$, respectively (Blietz et al. 1994). Assuming $T_{4}=1.2$ and $n_{e}=500$, which were derived from the mid-IR [Fe II $]$ lines (Rho et al. 2001), we estimate $N_{\mathrm{Fe}^{+}}$and thus the $\mathrm{Fe}^{+}$mass, $M_{\mathrm{Fe}^{+}}$, from the area of each region. As a result, $M_{\mathrm{Fe}^{+}}$is $5,1,0.9$, and $0.8 \times 10^{-4} M_{\odot}$ for regions A to D. Note that, if we adopt the typical values derived from the near-IR [Fe II] lines in SNRs, $T_{4}=0.5$

and $n_{e}=1 \times 10^{4}$ (e.g., Graham et al. 1987; Koo et al. 2007), $M_{\mathrm{Fe}^{+}}$decreases by an order 
of magnitude, while if we adopt the gas density of only a few times $10 \mathrm{~cm}^{-3}$ which was suggested by H I (Lee et al. 2008) and optical line observations (Fesen \& Kirshner 1980), $M_{\mathrm{Fe}^{+}}$increases by an order of magnitude. Accordingly the above $\mathrm{Fe}^{+}$masses can have such systematic errors.

On the other hand, by assuming the dust absorption coefficient given by Hildebrand (1983), a grain radius of $0.1 \mu \mathrm{m}$, and a specific dust mass density of $3 \mathrm{~g} \mathrm{~cm}^{-3}$, the mass of the warm dust, $M_{\mathrm{d}}$, is expressed as

$$
M_{\mathrm{d}}=10^{4}\left(\frac{L_{\mathrm{warm}}}{10^{8} L_{\odot}}\right)\left(\frac{T_{\text {warm }}}{40 \mathrm{~K}}\right)^{-5} M_{\odot},
$$

where $T_{\text {warm }}$ and $L_{\text {warm }}$ are the temperature and luminosity of the warm dust, respectively, derived from the above SED fitting. As a result, we obtain $M_{\mathrm{d}}$ of 50, 2, 0.9, and 50 $\times 10^{-4} M_{\odot}$ for regions A to D. Hence the $M_{\mathrm{Fe}^{+}} / M_{\mathrm{d}}$ ratios are $0.1,0.5,1$, and 0.02 for regions A to D. To first order, the absorption coefficient of a silicate grain is proportional to its volume, and thus the mass estimation is not affected by the grain size, although the shock processing of dust will change the grain size distribution (e.g., Andersen et al. 2011). If stochastic heating of very small grains is dominant, the dust masses could be overestimated.

The above huge differences in $M_{\mathrm{Fe}^{+}} / M_{\mathrm{d}}$ can be interpreted by the differences in the ratio of the destroyed to the non-destroyed dust mass from region to region. We estimate the latter ratios to be $30,70,80$, and $8 \%$ for regions $\mathrm{A}$ to $\mathrm{D}$, assuming that all elements in dust are removed away by sputtering at the same rate, all gas-phase Fe is singly ionized, and Fe initially depleted to dust accounts for $22 \%$ of $M_{\mathrm{d}}$ (Draine et al. 2007). Accordingly, the initial dust masses are $70,7,5$ and $50 \times 10^{-4} M_{\odot}$ for regions A to D. The difference between regions $\mathrm{A}$ and $\mathrm{D}$ can be explained by the fact that more destructive shock is propagating in region $\mathrm{A}$ than in region $\mathrm{D}$ where the deceleration of the shock due to the molecular cloud is indicated (Rho et al. 2001). Our result shows that the spatial distribution of the initial 
dust grains is highly biased toward regions A and D, probably reflecting the distribution of the pre-existing ISM.

In regions $\mathrm{B}$ and $\mathrm{C}$, the dust seems to be almost completely destroyed, which might be reasonable considering that the shock front reached the interior regions earlier. The sputtering destruction time of dust in the hot plasma of IC 443 is estimated to be $\sim 1 \times 10^{5}$ yr (Draine \& Salpeter 1979), by using the plasma density and temperature of $1 \mathrm{~cm}^{-3}$ and $10^{7} \mathrm{~K}$ (Petre et al. 1988), respectively, and assuming the initial grain radius of $0.1 \mu \mathrm{m}$. This time scale is, however, considerably longer than the age of IC 443. Jones et al. (1994) showed that dust destruction associated with a J-shock is much more efficient, which is suitable for regions A and D, and could also be viable for regions B and C. On the other hand, the time for Fe to reach an ionization equilibrium in hot plasma, $\sim 10^{12} n_{e}^{-1} \mathrm{~s}$, where $n_{e}$ is the electron density of X-ray plasma (Masai 1994), is estimated to be $\sim 2 \times 10^{4}$ yr, by using $n_{e}$ of $1.7 \mathrm{~cm}^{-3}$ obtained for IC 443 by Yamaguchi et al. (2009). Since this time scale is comparable to the age of IC 443, a significant fraction of gas-phase Fe atoms is likely to be more highly ionized than $\mathrm{Fe}^{+}$in regions $\mathrm{B}$ and $\mathrm{C}$, whereby we may underestimate the $\mathrm{Fe}^{+}$masses (i.e., the destroyed dust masses) there.

The $\mathrm{Fe}^{+}$mass in the central region of IC 443 is estimated to be $2 \times 10^{-3} M_{\odot}$ from the total [Fe II] line flux in the observed area relatively free of the pre-existing ISM, where the intensity of the warm dust emission is lower than $1.5 \times 10^{-3} \mathrm{ergs} \mathrm{s}^{-1} \mathrm{~cm}^{-2} \mathrm{sr}^{-1}$ (i.e., the lowest contour level in the right panel of Fig. 2). According to the model calculation, the ${ }^{56} \mathrm{Fe}$ mass produced from a $15-25 M_{\odot}$ core-collapse supernova is $0.05-0.13 M_{\odot}$ (Thielemann et al. 1996), which is one to two orders of magnitude larger than the $\mathrm{Fe}^{+}$ mass derived from our estimation. Thus our result suggests that Fe ejecta may not be so abundant as predicted theoretically for a core-collapse supernova. 


\section{Conclusion}

With the IRSF/SIRIUS, we observed the Galactic SNR IC 443, using the narrow-band filters for the [Fe II] $1.257 \mu \mathrm{m}$ and $1.644 \mu \mathrm{m}$ lines. Our observations detected the [Fe II] line emission from a wide area of IC 443, not only the shock-heated shell regions, but also interior regions, with prominent filamentary, shell-like structures. From a combination of the [Fe II] $1.257 \mu \mathrm{m}$ and $1.644 \mu \mathrm{m}$ lines which are attributed to electronic transitions from the same upper energy level, we estimated the foreground extinction and obtained extinction-corrected [Fe II] line maps. We also obtained the map of the shock-heated warm dust emission using $A K A R I$ and Spitzer IR images. As a result, we find that the [Fe II] line emission is notably strong relative to the warm dust emission in the inner central part of IC 443. We estimated the $\mathrm{Fe}^{+}$masses from the intensity of [Fe II] line and compared them with the masses of the shock-heated warm dust, which are derived from the SED fitting by using the $A K A R I$ and Spitzer IR images. The result implies that the mass ratios of $\mathrm{Fe}^{+}$to the dust are 0.1 (ionic shock shell), 0.02 (molecular shock shell), and 0.5 and 1 (interior regions). The large difference in the ratio likely reflects that in the dust destruction efficiency; in particular, the $\mathrm{Fe}^{+} /$dust enhancement in the interior regions requires an efficient destruction mechanism for large grains, or contribution of theoretically-predicted Fe ejecta.

We thank the anonymous referee for giving us helpful comments to improve the paper.

The IRSF project was financially supported by the Sumitomo foundation and Grants-in-Aid for Scientific Research on Priority Areas (A) (Nos. 10147207 and 10147214) from the Ministry of Education, Culture, Sports, Science and Technology (MEXT). The operation of IRSF is supported by Joint Development Research of National Astronomical Observatory of Japan, and Optical Near-Infrared Astronomy Inter-University Cooperation Program, funded by the MEXT of Japan. This research is based on observations made with $A K A R I$, 
a JAXA project with the participation of ESA, and with Spitzer, which is operated by the Jet Propulsion Laboratory, California Institute of Technology under a contract with NASA. 


\section{REFERENCES}

Andersen, M., Rho, J., Reach, W. T., Hewitt, J. W., \& Bernard, J. P. 2011, ApJ, 742, 7

Bertin, E., \& Arnouts, S. 1996, A\&AS, 117, 393

Blietz, M., Cameron, M., Drapatz, S., et al. 1994, ApJ, 421, 92

Bykov, A. M., Krassilchtchikov, A. M., Uvarov, Y. A., et al. 2008, ApJ, 676, 1050

Cardelli, J. A., Clayton, G. C., \& Mathis, J. S. 1989, ApJ, 345, 245

Castelletti, G., Dubner, G., Clarke, T., \& Kassim, N. E. 2011, A\&A, 534, A21

Doi, Y., Komugi, S., Kawada, M., et al. 2012, PKAS, 27, 111

Draine, B. T., \& Salpeter, E. E. 1979, ApJ, 231, 77

Draine, B. T. 1995, Ap\&SS, 233, 111

Draine, B. T., Dale, D. A., Bendo, G., et al. 2007, ApJ, 663, 866

Fesen, R. A., \& Kirshner, R. P. 1980, ApJ, 242, 1023

Gaensler, B. M., Chatterjee, S., Slane, P. O., et al. 2006, ApJ, 648, 1037

Graham, J. R., Wright, G. S., \& Longmore, A. J. 1987, ApJ, 313, 847

Hildebrand, R. H. 1983, QJRAS, 24, 267

Jones, A. P., Tielens, A. G. G. M., Hollenbach, D. J., \& McKee, C. F. 1994, ApJ, 433, 797

Kawada, M., Baba, H., Barthel, P. D., et al. 2007, PASJ, 59, 389

Koo, B.-C., Moon, D.-S., Lee, H.-G., Lee, J.-J., \& Matthews, K. 2007, ApJ, 657, 308

Lee, J.-J., Koo, B.-C., Yun, M. S., et al. 2008, AJ, 135, 796 
Lee, J.-J., Koo, B.-C., Snell, R. L., et al. 2012, ApJ, 749, 34

Masai, K. 1994, ApJ, 437, 770

Nagashima, C., Nagayama, T., Nakajima, Y., et al. 1999, in Star Formation 1999, ed. T. Nakamoto (Nagano: Nobeyama Radio Obs.), 397

Nagayama, T., Nagashima, C., Nakajima, Y., et al. 2003, Proc. SPIE, 4841, 459

Noriega-Crespo, A., Hines, D. C., Gordon, K., et al. 2009, The Evolving ISM in the Milky Way and Nearby Galaxies

Nussbaumer, H., \& Storey, P. J. 1988, A\&A, 193, 327

Olbert, C. M., Clearfield, C. R., Williams, N. E., Keohane, J. W., \& Frail, D. A. 2001, ApJ, 554, L205

Petre, R., Szymkowiak, A. E., Seward, F. D., \& Willingale, R. 1988, ApJ, 335, 215

Rieke, G. H., Young, E. T., Engelbracht, C. W., et al. 2004, ApJS, 154, 25

Rho, J., Jarrett, T. H., Cutri, R. M., \& Reach, W. T. 2001, ApJ, 547, 885

Shinn, J.-H., Koo, B.-C., Seon, K.-I., \& Lee, H.-G. 2011, ApJ, 732, 124

Skrutskie, M. F., Cutri, R. M., Stiening, R., et al. 2006, AJ, 131, 1163

Thielemann, F.-K., Nomoto, K., \& Hashimoto, M.-A. 1996, ApJ, 460, 408

Troja, E., Bocchino, F., \& Reale, F. 2006, ApJ, 649, 258

Troja, E., Bocchino, F., Miceli, M., \& Reale, F. 2008, A\&A, 485, 777

Welsh, B. Y., \& Sallmen, S. 2003, A\&A, 408, 545

Yamaguchi, H., Ozawa, M., Koyama, K., et al. 2009, ApJ, 705, L6 
Yamamura, I., Makiuti, S., Ikeda, N., et al. 2009, in ASP Conf. Ser. 418, AKARI, a Light to Illuminate the Misty Universe, ed. T. Onaka et al. (San Francisco, CA: ASP), 3 
Table 1. Summary of the present IRSF observations

\begin{tabular}{cccccc}
$\lambda_{\text {center }}(\mu \mathrm{m})$ & Transition & $\Delta \lambda_{\text {eff }^{\mathrm{a}}}(\mu \mathrm{m})$ & Exposure $(\mathrm{s})$ & NDI $^{\mathrm{b}}$ & Date \\
\hline 1.257 & $a^{4} D_{7 / 2} \rightarrow a^{6} D_{9 / 2}$ & 0.028 & 60 & 10 & Nov 2012 \\
1.644 & $a^{4} D_{7 / 2} \rightarrow a^{4} F_{9 / 2}$ & 0.018 & 30 & 15 & Feb, Nov 2012 \\
\hline
\end{tabular}

${ }^{a}$ Effective band width calculated from $\int S(\lambda) d \lambda=T_{\lambda} \Delta \lambda_{\text {eff }}$, where $\mathrm{S}(\lambda)$ is a filter response curve, and $T_{\lambda}$ is a throughput at $\lambda_{\text {center }}$.

${ }^{\mathrm{b}}$ Number of dithered images.

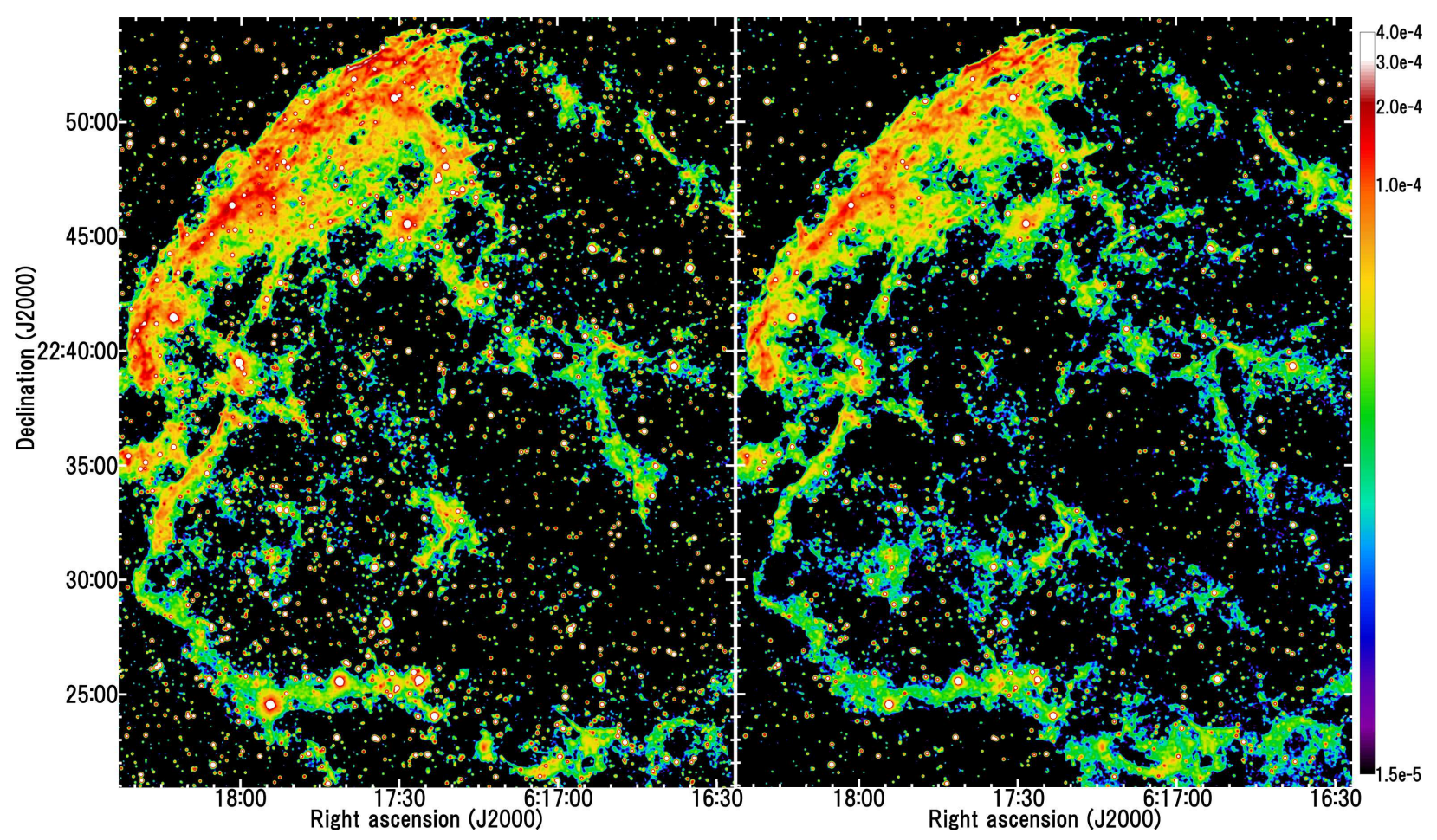

Fig. 1.- [Fe II] $1.257 \mu \mathrm{m}$ (left) and $1.644 \mu \mathrm{m}$ (right) line maps, smoothed with a Gaussian kernel of $2.3^{\prime \prime}$ in sigma. The color levels are given in units of $\operatorname{ergs~s}^{-1} \mathrm{~cm}^{-2} \mathrm{sr}^{-1}$. 

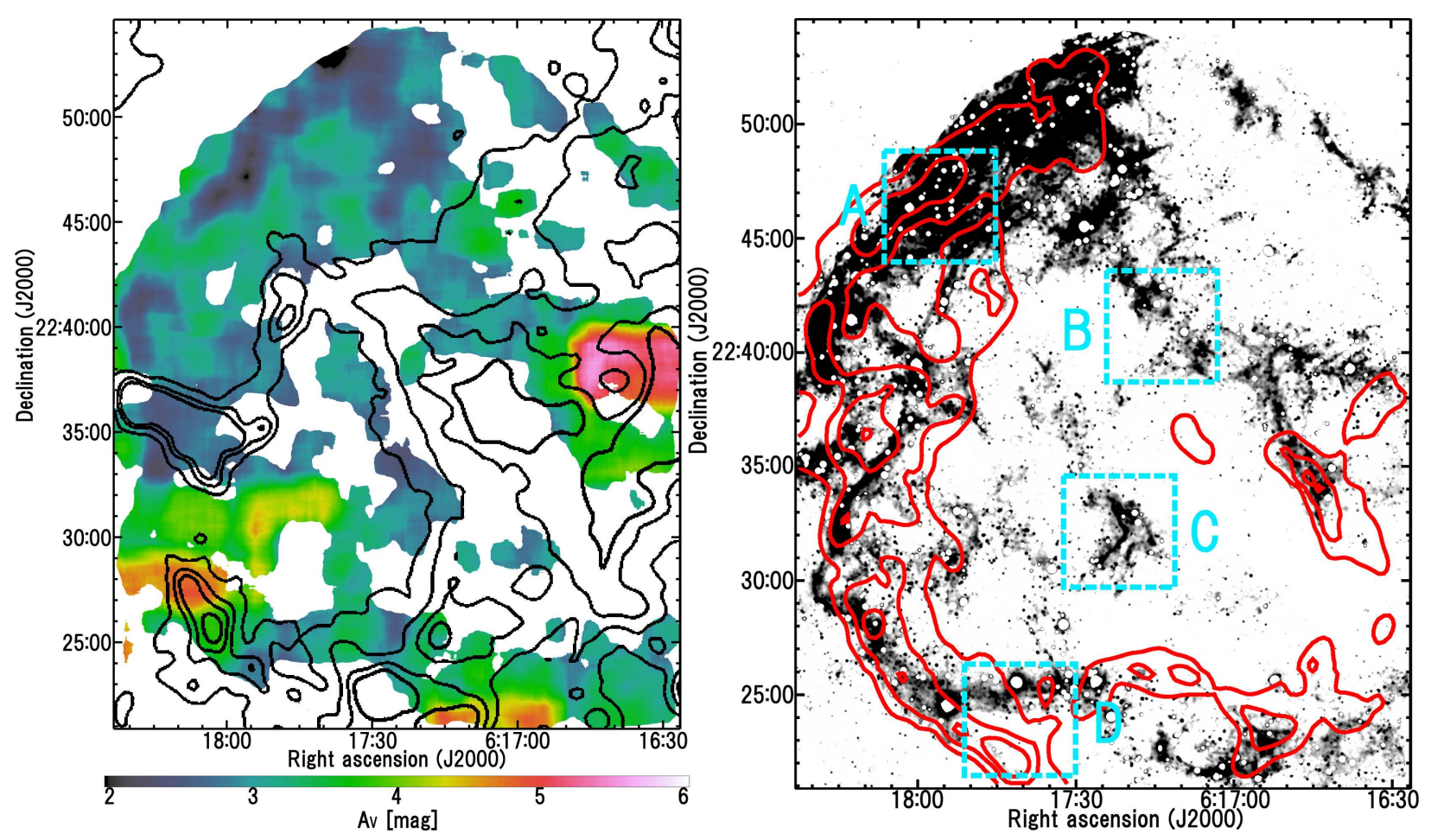

Fig. 2.- (Left) Foreground dust extinction $A_{V}$ map derived from the observed [Fe II] line intensities, with the contours of the ${ }^{12} \mathrm{CO}$ map integrated in the velocity range of -10 to 0 $\mathrm{km} \mathrm{s}^{-1}$ taken from Lee et al. (2012). (Right) Spatial distribution of the extinction-corrected [Fe II] $1.257 \mu \mathrm{m}$ line intensity, with the contours of the intensity of the warm dust emission integrated over the wavelength range of $2-200 \mu \mathrm{m}$, which is derived from the SED fitting. The gray scale ranges from 2.0 to $8.0 \times 10^{-5} \mathrm{ergs} \mathrm{s}^{-1} \mathrm{~cm}^{-2} \mathrm{sr}^{-1}$, while the contour levels are $1.5,2.5$, and $3.5 \times 10^{-3} \operatorname{ergs~s}^{-1} \mathrm{~cm}^{-2} \mathrm{sr}^{-1}$. 


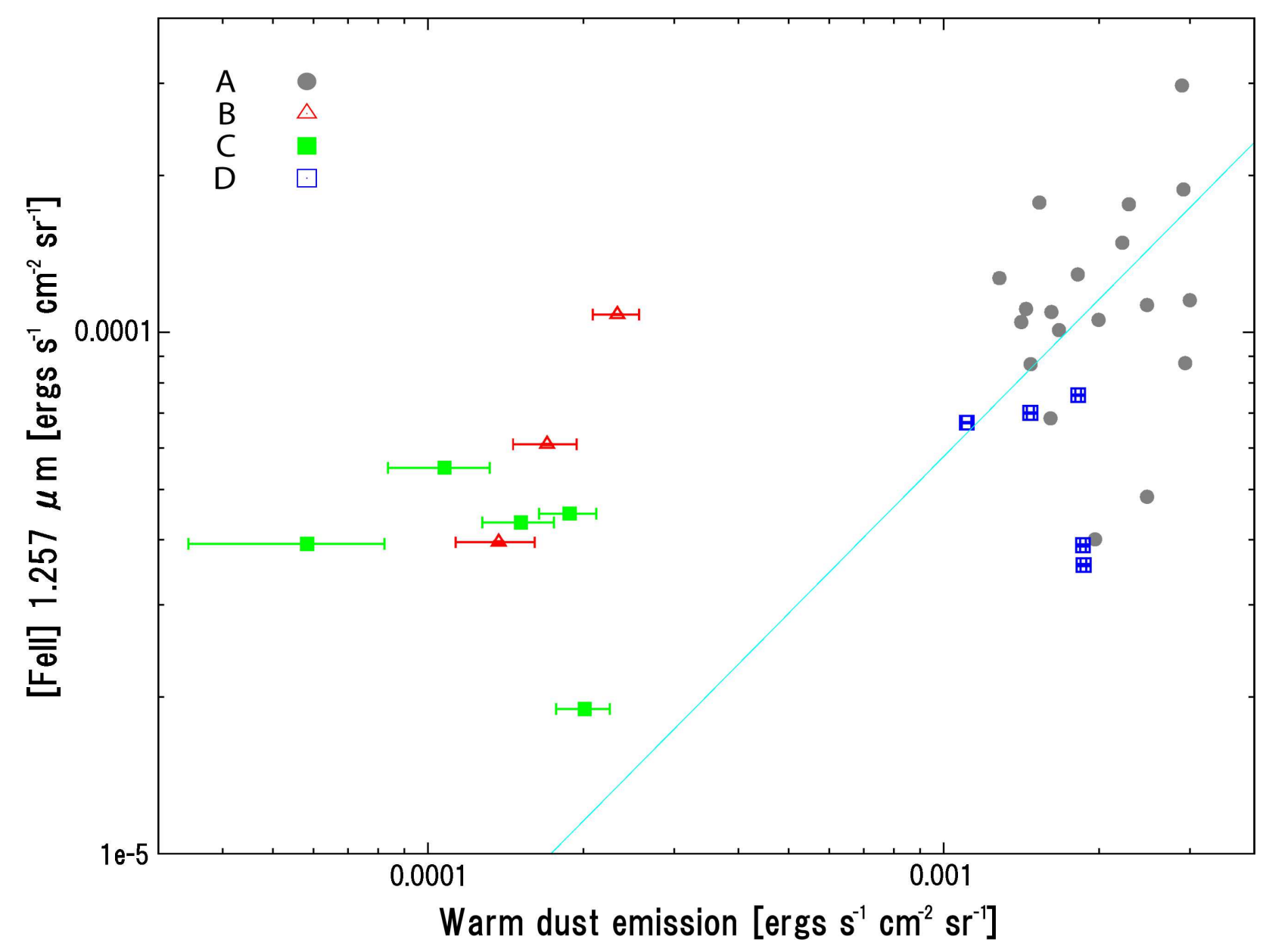

Fig. 3.- Correlation plot between the intensities of the extinction-corrected [Fe II] 1.257 $\mu \mathrm{m}$ line and the warm dust emission integrated over the wavelength range of $2-200 \mu \mathrm{m}$. Regions A to D are defined in Fig. 2, and the line fitted to only the data points of region A is overlaid. The data are spatially sampled every $1^{\prime}$, which corresponds to 130 pixels of the camera array. 